Culture et histoire dans l'espace roman

$6 \mid 2011$

Figures du pouvoir dans la littérature hispanoaméricaine

\title{
La chambre noire du sens
}

Représentations du pouvoir dans Muertos incómodos du Subcomandante Marcos et Paco Ignacio Taibo II

\section{Nathalie Galland}

\section{OpenEdition}

\section{Journals}

Édition électronique

URL : https://journals.openedition.org/cher/9600

DOI : $10.4000 /$ cher.9600

ISSN : 2803-5992

Éditeur

Presses universitaires de Strasbourg

Édition imprimée

Date de publication : 30 juin 2011

Pagination : 115-126

ISBN : 978-2-35410-033-9

ISSN : 1968-035X

Référence électronique

Nathalie Galland, «La chambre noire du sens », reCHERches [En ligne], 6 | 2011, mis en ligne le 17 décembre 2021, consulté le 26 janvier 2022. URL : http://journals.openedition.org/cher/9600 ; DOI https://doi.org/10.4000/cher.9600

\section{(c) (i) 8 (2)}

Ce(tte) œuvre est mise à disposition selon les termes de la Licence Creative Commons Attribution -

Pas d'Utilisation Commerciale - Partage dans les Mêmes Conditions 4.0 International. 


\title{
La chambre noire du sens. Représentations du pouvoir dans Muertos incómodos du Subcomandante Marcos et Paco Ignacio Taibo II
}

\author{
NATHALIE GALLAND \\ Université de Bourgogne
}

\begin{abstract}
Es sabido que el asesino siempre regresa a la escena del crimen. Pero supongamos que el Elías y el Belascoarán no van detrás de un asesino, sino de EL asesino. Si es quien yo me imagino, entonces EL asesino no va a regresar a la escena del crimen, simple y sencillamente porque él es la escena del crimen. EL asesino es el sistema. El sistema sí. Cuando hay un crimen hay que buscar al culpable arriba, no abajo. El Mal es el sistema y los Malos son quienes están al servicio del sistema ${ }^{1}$.
\end{abstract}

\begin{abstract}
$\mathrm{C}$ 'est le système l'assassin! C'est le système l'assassin qui farde sous l'apparence du légitime l'arbitraire et la corruption, qui «donne bonne figure au cadavre, [...], transforme l'ordre en parure, le cosmos en cosmétique» (Derrida: 177). Dans ce polar oblique écrit à quatre mains par le Subcomandante Marcos et Paco Ignacio Taibo II, s'élabore la
\end{abstract}

1 Muertos incómodos (falta lo que falta), México, Joaquín Mortiz, 2005, p. 53. Le roman, écrit par chapitres publiés initialement par le supplément dominical de La Jornada en 2004 - chapitres impairs écrits par Marcos, chapitres pairs et épilogue par Taibo II met en scène deux enquêteurs. De la forêt lacandone, Elías Contreras est envoyé par le commandement militaire de l'Ejército Zapatista de Liberación Nacional à Mexico pour suivre la trace d'un dénommé Morales, impliqué dans diverses opérations criminelles en forêt lacandone et territoire zapatiste. Point d'importance, Elías fait partie lui-même de ces muertos incómodos, los muertos de abajo que nunca se están quietos. Dans le même temps, à México, le fameux détective Héctor Belascoarán Shayne mène lui aussi une enquête : son client reçoit des coups de fil énigmatiques d'un mort, ancien militant étudiant en 68 et assassiné par le pouvoir en 71, propos d'outre-tombe donc, qui construisent peu à peu, par le jeu de révélations parfois spectaculaires, le portrait d'un autre Morales. 
représentation d'un pouvoir mexicain saisi dans son illégitimité, un pouvoir mexicain objet lui-même de bien des pouvoirs, celui du voisin gringo, celui de l'argent sale, celui des multiples réseaux d'influence. Un pouvoir dès lors symptomatique de la décomposition d'un système, dont en réalité le roman propose une autopsie loufoque.

Le roman semble faire d'abord l'épreuve du réel représenté, se frotter délibérément au pourrissement d'un monde qui, pareil à la charogne de Baudelaire, s'agite, remue, ondule jusqu'à séduire, mais ne constitue plus qu'un corps inerte, parce qu'orphelin de toute valeur éthique. Les représentations du pouvoir construites au fil des pages demeurent toujours en prise directe avec la violence d'état et les multiples formes de l'antipolitique, déterminant ainsi la frontière exemplaire entre le bien et le mal. $\mathrm{Si}$, pour Walter Benjamin, l'Histoire se réfracte non pas en histoires mais en images, précisément celle du bien et du mal, pour manichéenne qu'elle soit, constituera la perspective depuis laquelle s'énonce la déchirure du réel, la distorsion du lien social et le morcellement de la mémoire, mais aussi l'oppression, la répression, la torture, l'injustice, le manque.

Faire l'épreuve du réel dans les mots, c'est changer le texte en un laboratoire de formes et de sens, c'est faire de l'espace du roman le bain capable de révéler la nature du pouvoir, capable de le défaire de ses artifices dans des combinaisons fictionnelles décapantes. De trompe-l'oeil en trompe-leurres, de cryptages en décryptages en recryptages, le roman s'attache parallèlement à défaire les illusions d'une société par trop suiviste, et comme dans une chambre noire, fidèle en cela au genre policier, donne bien à voir l'envers du monde, la «vraie» face du pouvoir.

\section{Ben laden, Hollywood et la géopolitique}

\section{De la prestidigitation}

Parmi les représentations récurrentes du pouvoir mexicain, s'énonce assez vite dans le roman, celle d'un pouvoir lui-même objet de pouvoir. Dans des mises en scènes échevelées, se joue la subordination la plus exemplaire du pouvoir mexicain à la stratégie politique, à la fascinante modernité et au néolibéralisme des États-Unis. Nombre de voix fictionnelles dénoncent ainsi la figure gringa à coups, parfois, de références historiques qui viennent légitimer une indignation, marque indirecte d'une lutte pour la dignité. Dans ces lignes, le Mexique apparaît comme un territoire vide de toute autorité, un espace à saisir dans une salle des ventes internationale... Quand le processus 
historique rappelé est celui de la subordination, de la dépossession. Là, le roman va en faire des tonnes, va mettre en tension sa condition générique de roman noir, même parodique et hétérodoxe, et l'infusion du capitalisme sauvage au cœur du pouvoir: se saisissant de la situation de domination économiquement et politiquement vérifiable, il va entrer lui-même dans une foire aux enchères du non-sens; surenchérir, caricaturant à l'extrême le rapport de soumission et de manipulation. Ainsi en est-il par exemple du pillage organisé de la cité des dieux, de Teotihuacán. Une cité qui, dans le roman, serait concrètement démantelée pierre par pierre par la Wal-Mart (l'immense et très puissant groupe commercial et financier nord-américain) et progressivement remplacée par une copie conforme en carton-pâte. La domination de la société de consommation servant désormais au pillage du patrimoine culturel, dans une entreprise de «disneyisation» du monde où les vessies bientôt deviendraient des lanternes. Mais précisément, l'image de la pyramide, c'est la figure d'un Mexique mythique où s'incarne un Age d'or sacralisé ; la figure d'un pouvoir transféré ici petit à petit de l'autre côté du fleuve... Et politiquement, la pyramide est aussi l'édifice métaphorique du gouvernement autoritaire: un édifice "dont le foyer serait le sommet de la pyramide aussi bien que la source transcendante d'autorité au-dessus de lui» (Arendt 1972). Définition miroitante pour le système du pouvoir au Mexique, conformément à la réalité fictionnelle au moins: au sommet de la pyramide, le système passablement corrompu du pouvoir, au-delà, comme force et entité transcendantale tutélaire, le modèle des États-Unis, la domination idéologique, économique et culturelle...

L'élaboration sémantique à l'œuvre dans le roman met en évidence une praxis qui ne répond plus au bien commun, mais à un alignement stratégique sur le véritable pouvoir dominant le pouvoir, par l'interchangeabilité du réel et de son double. L'on entre dès lors dans un fac-similé du monde où le Mexique n'est plus que fantomatique extension des États-Unis, un souscontinent à l'autonomie fictive, réservoir de main d'œuvre, d'objets d'art exotiques et de ressources naturelles. Cette pratique politique du trompel'œil, du leurre, irrigue le roman dès lors que, depuis ce Mexique servile et avili, sont convoquées les représentations du pouvoir états-unien: quand la manipulation devient la norme de gouvernance, l'axe majeur d'une géopolitique plus proche des scénarios hollywoodiens, de leurs décors et de leurs effets spéciaux, que de la pratique diplomatique.

Extraordinairement éloquent encore le fragment où l'informateur d'Héctor Belascoarán lève le voile sur la véritable identité de Ben Laden. 
Le décor - la tente dans le désert - et le personnage lui-même - barbu et enturbanné - ne serait que le produit d'un montage vidéo réalisé dans les studios douteux de Burbank en Californie, à la demande du gouvernement Bush. Concrètement, on aurait recyclé un ex-vendeur de tacos de Ciudad Juárez, dénommé Juancho, recyclé pour moitié dans le clip politique pour moitié dans le cinéma pornographique, le taquero en question passant d'un studio de tournage à l'autre, en changeant simplement de masques, passant de Ben Laden à El Horrible, super héros déguisé en démon de la lucha libre, dès lors engagé dans d'autres corps à corps...

Le pouvoir qui téléguide la praxis politique mexicaine, est un pouvoir qui organise le formidable règne du faux-semblant où le réel se réfracte dans la matière et les scénarios fictifs, dans l'artifice qui garantit l'apparence du même. Le masque ainsi se fait écran, endroit d'une dissimulation et envers pour une projection, supercherie et cynisme à l'appui, un modus operandi inspirant pour un pouvoir mexicain prisonnier volontaire d'une certaine mimèsis:

El país era un gran negocio, un territorio convertido en botín por jinetes apocalípticos chagas y medio narcos; un supermercado gerenteado por un Federico Nietzche (sic) pedo, muy pedo, donde nada era lo que parecía. Era como una telenovela venezolana con Alí Babá de secundario y los 40 ladrones de estelares (69).

C'est d'abord en rupture avec cet habitus ${ }^{2}$ de la corruption du pouvoir que se tisse un discours de l'oblique essentiellement parodique, en rupture radicale avec une langue de bois pratiquée par une classe dirigeante réduite à sa propre pérennité. La fragmentation du monde, schisme entre l'être et le réel, entre le pouvoir et l'autorité, est alors soumise à la fragmentation de l'écriture. Cette volonté d'une résistance du dire au voir, passe par le choix d'une expression travaillée dans sa matière et sa structure, sa méthode et son rythme, quand le récit semble pousser la langue dans des retranchements essentiellement obliques. Ainsi s'offre la langue d'Elías, dans son observation des tours de passe-passe politiques:

y entonces yo no muy le entendí y él me explicó que él hace que la gente mire una mano y ya con la otra mano esconde o saca lo que tiene escondido. Y entonces yo le pregunté si es como hacen los políticos que te ponen a mirar

2 Selon Pierre Bourdieu: «des habitus, systèmes de dispositions durables et transposables, structures structurées prédisposées à fonctionner comme structures structurantes, c'est-àdire en tant que principes générateurs et organisateurs de pratiques et de représentations qui peuvent être objectivement adaptées à leur but sans supposer la visée consciente de fins et la maîtrise expresse des opérations nécessaires pour les atteindre [...]» (88). 
una cosa mientras por otro lado están haciendo sus maldades. Y entonces el Alakazam me dijo que eso mero, pero que los políticos no eran magos sino que eran unos hijos de puta, así dijo (191).

Ou encore: "Y entonces la problema [sic] es que no sólo se trata de los que hicieron sus maldades en aquellos años del gobierno del Zedillo, sino que todavía por ahí andan los culpables y todavía se sigue haciendo lo mismo... » (197). Lui qui fait récit en étant mort, semble tout indiqué pour capturer les distorsions ordinaires du réel, mettre en abîme dialectiquement l'obliquité du monde dans celle de la langue. Portée par la transgression lexicale et les traces chiapanèques d'une oralité paysanne et parfois indigène (double possessif notamment), sa voix narrative singulière compose la preuve linguistique d'un hiatus sémantique inhérent à la praxis anti-politique du pouvoir. Mise à distance par ce regard, la matière carnavalesque du réel engagée dans la prestidigitation à grande échelle succombe à un déchiffrage poétique, quand c'est la métaphore qui livre la vérité des choses. Quand l'élaboration narrative fait jaillir une nouvelle pertinence sémantique ancrée dans une impertinence prédicative capable de déclôturer le cadre du dire.

Qu'il utilise l'effet loupe ou la perspective gigogne, le roman creuse la discordance pour produire un renversement de l'image, tel qu'il a lieu dans les chambres noires photographiques. Dans ses différents déploiements, le pouvoir est à son tour placé dans la camera obscura de l'oblique où se produit l'inversion de l'inversion. Refusant le simple donné historique mais considérant l'Histoire comme un processus non figé sur lequel l'on peut dès lors agir, l'oblique est acte po-éthique, acte politique.

\section{Le monstre : asphalte, violence et défiguration \\ De l'oubli}

Le Subcomandante Marcos qui intervient dans le récit comme dirigeant de l'EZLN, a prévenu Elías: "Acuérdate que a la ciudad de México le decimos "el Monstruo" ». Bientôt en effet, la ville de México devient territoire métonymique de la violence et de la corruption, labyrinthe réinventé, sans centre et donc sans issue, où s'exercent tous les abus de pouvoir. S'il n'est pas surprenant qu'un roman noir plante le décor de l'enquête dans les basfonds urbains, et si le $D F$ constitue bien l'espace exemplaire, où se nouent les exactions, ce centre s'expose fondamentalement dans le décentrement le plus cru de l'illégitimité. Il n'est plus, loin s'en faut, l'en-droit du logos, mais celui au contraire d'une Béotie perpétuelle, lieu des décombres de la violence 
sociale et de la violence d'État, espace propice à la défiguration. C'est que ce pouvoir, déraisonnable en tout point, c'est-à-dire hors de toute mesure et de toute vertu, ne limite plus son arbitraire et s'incarne dans l'espace comme le tyran dans l'histoire, comme un "grand individu violent", selon le terme ricœurien. Défiguration donc, défiguration des êtres d'abord et parmi eux, du détective qui confesse:

El ojo que me falta me lo voló un ex comandante de la judicial, hoy finado. Cojeo por culpa de un escopetazo que me metieron los mismos que organizaron los halcones. $\mathrm{Y}$ me he pasado siete meses y tres días en una cárcel en Tabasco por documentar un fraude electoral del PRI hace unos añitos (96).

Défiguration, c'est-à-dire production d'un autre visage, portrait littéralement pro-tractus qui projette le sens dans une étrange transhumance: défiguration, dissonance et dissidence du sujet. De fait, l'univers du détective est rendu par une langue ironique, qui, dans la distinction des espaces de production du discours et de l'expression même, s'attaque à la hiérarchie des valeurs et joue sur le renversement du monde. Morsure au vitriol, issue de la part acide et ludique du langage, elle transforme l'espace du roman en lieu d'un dissensus:

Ce qu'expose la littérature, c'est cette expérience de la multiplicité et du dissensus, cette expérience de "l'ordinaire-extraordinaire" qui vient en n'importe quel point trouer le consensus. [...] Le propre-impropre de la littérature, ce qui la lie à la démocratie, ne serait-ce pas alors [qu'elle] ne cesse d'inscrire l'expérience du quasi-autre et du dissensus, l'expérience de la multiplication vertigineuse du banal qui parle et se dérobe, du banal extraordinaire? (Rancière 2004: 196-197).

Et ce dissensus qui interroge la démocratie comme il l'invoque, qui déchire l'uniforme peau du monde pour y surgir, constitue l'arme capable de mettre en déroute la violence structurée et structurante du pouvoir et sa cohorte de laquais. Défiguration des protagonistes donc et aussi dégradation de l'espace, métaphore de la perte, du déséquilibre du monde aux confins du monde qu'incarne cette ville tentaculaire et transgressive, poulpe géant, monstrueuse oui, quand la figure produite semble s'inscrire dans une sorte de darwinisme à l'envers, un darwinisme de figures au bout du compte défigurées par l'évolution. Défiguration, déprédation du sens aussi: «En los últimos años había vivido en las fronteras, en el límite, de unos extraños territorios que bordeaban la incoherencia, la irracionalidad y la extravagancia, y también la tragedia, la pendejez, el agravio colectivo, la impunidad, el miedo y el ridículo» (69). 
Rien d'étonnant alors à voir ce réel disséqué par la double enquête d'un borgne, aguerri aux rites de la mégapole et amateur d'humour noir, et d'un mort, dont le regard et la langue semblent pouvoir capturer les multiples figures spectrales du pouvoir. L'univers urbain saisi par la marginalité des regards est celui des petits et grands arrangements avec la violence, l'incontrôlable dérive du corps urbain. Surgissent donc des voix minuscules, victimes directes ou indirectes de l'altération du système, les faux chauffeurs de taxi, les selocuidos, les joueurs de domino, et dans leurs voix, s'égrènent les affaires d'état, les meurtres d'opposants politiques, la corruption des fonctionnaires à tous les niveaux du système, surtout les plus hauts. Une infinité d'abus de pouvoir et d'exactions rendue par le procédé de l'ajout, de la liste, procédé capable de représenter la fugue, l'extraordinaire ligne de fuite empruntée par le pouvoir, procédé susceptible aussi d'épuiser dans la véracité des mots, la voracité du réel: «Y entonces dijo que ahí había de todo, que sea represión, asesinatos, cárceles, perseguidos, desaparecidos, fraudes, robos, despojos de tierras, venta de la soberanía nacional, traición a la Patria, corrupción» (172).

Par fragments, se compose un tableau radiographique du système de pouvoir en place au Mexique depuis 1968; date emblématique de l'exigence démocratique et de la violence d'état qui lui fit réponse, mutilation baignée par une temporalité du piétinement... Espace d'une érosion acide des choses et des êtres jusqu'à l'infra-humanité urbaine, México est aussi le lieu de l'effacement, de l'engouffrement dessous les réseaux souterrains et les connexions obscures. Bientôt, la capitale, "donde habita el olvido» se manifeste comme l'abîme où sont englouties la mémoire et l'auctoritas - cette vertu d'avant la loi et l'exercice du pouvoir - le lieu de l'abandon du sens: "No había nada que ver. Cajas y papeles. Las huellas habían desparacido. El archivo histórico se había comido a la memoria histórica, a la simple memoria» (100).

Se souvenir, c'est parcourir le territoire de miroirs liquides, passer de l'autre côté des subterfuges, exhumer, échapper au labyrinthe où s'exercent de multiples tensions, tensions des corps, des simulacres, du poids hégémonique... Dissoudre le recordar, c'est dès lors user du pouvoir comme de l'épanchement poisseux de l'amnésie; l'oubli est un autre masque, intimement lié au silence, un geste second imperturbable qui défigure à son tour la mémoire en lui ôtant toute corporéité, en la faisant intégrer l'espace de la virtualité: figure, transfiguration, défiguration et peut-être préfiguration d'une amnésie collective perdurable. Cet engloutissement, qui s'énonce au 
gré du territoire urbain, rime dès lors avec la disparition de l'autorité, parce que la mémoire - mémoire ordinaire de la résistance en tension avec la mémoire historique -, c'est aussi la garantie de la permanence de la légitimité des "assises du monde», de l'autorité. L'étymologie du terme est porteuse de sens : elle renvoie à l'auctor (l'agissant) et à l'idée d'augmentation, "augmentation de la puissance de déchiffrement du sens, de la capacité à l'harmonisation consensuelle de la société» (Rancière 2004). Ce qu'elle impose, renouvelle et amplifie, s'inscrit dans la légitimité et l'héritage.

Le pouvoir se fait dès lors à la fois assassin et victime, territoire de l'abus et corps violé, à la fois bourreau et gisant. Dans sa forme corrompue et violente, il occupe bien tout l'espace mais a gommé le temps en dérobant la mémoire. Seul le roman, parodique, certes loin d'être abouti en termes littéraires, pourtant se pense et s'offre comme une proposition, un initium désiré, la voie pour une re-fondation indirecte des valeurs du bien commun, retour d'un nouveau temps des origines, renouveau de l'autorité quand le pouvoir est "pouvoir des commencements", dimension du lien social, force liante du vivre ensemble des hommes.

\section{Terrorisme d'état et coups financiers en milieu tropical humide}

De la force

On l'aura bien compris: directement lié à une occupation de l'espace tout entière déployée dans la dégradation, l'exercice du pouvoir agit en prédateur du monde et des êtres qui ne veulent pas renoncer à l'éthique... Les mêmes causes produisant les mêmes effets, la selva Lacandone bientôt va se déployer comme l'espace de l'impunité et de l'exercice de la force entre autre coups fourrés, jungle allégorique de la sauvagerie, équivalent tropical humide au $D F$, transpirant de la même crasse...

Suivant le principe photographique qui fait du texte un agrandisseur d'images, c'est avec une référentialité maximale que vont être saisis les fragments les plus exemplaires d'un réel devenu objet de pouvoir... Monde sauvagisé des confins, selva et Altos du Chiapas se dessinent comme territoire où le donné historique de la marginalisation s'articule avec une géographie recentrée par la spéculation... Et d’abord la biosphère de Montes Azules, en réalité réservoir de bestioles exotiques exportables sous le manteau et de matières premières potentiellement lucratives: 
Zedillo y Carabias tienen negocios en Montes Azules. La ONG de Carabias es sólo una tapadera para el saqueo de especies animales, que colocan en varias partes del mundo por medio de una especie de mercado negro internacional. Lo de traficar con guacamayas, tapires, changos y otros animales que no me acuerdo ahora, es sólo el primer paso. En realidad, están preparando la entrada de grandes consorcios que van por la madera, el uranio y el agua. El agua será tan importante en este siglo como lo fue el petróleo en el pasado. Estoy hablando de dinero, mucho dinero. En el gabinete de Fox saben todo y se hacen patos (87).

Plus que contamination économique du politique, la perspective fictionnelle se resserre sur un véritable transfert d'un pouvoir exécutif élargi aux sphères économiques, quand la perte des valeurs éthiques s'accompagne d'un renversement hiérarchique, et coïncide, dans la matérialité du monde, avec une marchandisation générale organisée par le terrorisme d'état:

AUTORIDAD: ¿Qué cosa es El Yunque?

EL NO TAL MORALES: Es una organización clandestina de derecha, de extrema derecha, son los que ahorita están metidos en el PAN y en el gobierno de Fox. Y también están metidos en otros partidos políticos. En esa organización hay políticos, empresarios, obispos. Llevan años siguiendo con las enseñanzas de sus antecesores, o sea de Salinas y Zedillo. El plan es vender todo lo que se pueda vender y hacerse ricos. No les importa nada, ni la Patria ni la religión ni la gente, aunque digan lo contrario (215).

Monde sauvagisé aux confins, selva et Altos constituent le lieu d'exercice de la force, c'est-à-dire de la violence illégitime, qui n'observe d'autre règle que celle qui répond à sa volonté et à son intérêt. Là, rébellion et résistance zapatistes sont des entités à éliminer via la guerra sucia en vigueur depuis des décennies à coup de déplacements, déchirures des liens communautaires et répressions physiques; là se perpétue dans le silence officiel, le massacre d'Acteal le 22 décembre 1997, symptomatique de la violence extrême et de l'impunité des groupes paramilitaires...

Se ve que hay un acuerdo de los malos gobiernos para chingar a los pueblos indígenas zapatistas por medio de lo que se llama guerra sucia que quiere decir que es una guerra así como escondida, que no se muestra, que sea que hacen como que todo está bien pero no está bien, sino que hay muertos y desaparecidos y desplazados y muchas desgracias para los jodidos (196-197).

Pourtant, au bout du processus de la révélation photographique, sous ces latitudes où se déclinent les multiples spectres de la pratique anti-politique, s'énoncera bien la justice de ceux d'en bas, portée par les acteurs de l'inversion, du retournement des clichés ordinaires et des visions du monde, 
du retournement de la pyramide. Elías, Belascoarán, mais aussi la Magdalena, travelo romantique exporté du bitume defeño aux parages d'Ocosingo, tous useront de la solidarité pour détravestir le monde, construisant à contre-jour un univers de contrechamps et d'hyperfocalisations, refigurant radicalement les rapports sociaux, les modes du faire par les modes du dire.

\section{Éléments pour une conclusion}

Paul Ricoeur le soutient: "C'est, semble-t-il, le rôle de la plus grande partie de notre littérature de détruire le monde» (113). Depuis le territoire échevelé du texte, s'engage à l'évidence le démantèlement de morceaux de réel prélevés d'un corps politique rongé par la corruption. Ce corps politique qui a perdu tout socle et tout contour éthique, a fait de l'espace le territoire où peut à l'infini se répéter le même - spoliation, violence physique, exclusion -, se répéter un temps où autrefois s'originait l'autorité, mais qui n'est plus que piétinement, reproduction de masques, espace de l'agir d'un pouvoir réduit à la promesse du pire. Là, paraissent des témoins, résistants, militants étudiants de 1968, responsables syndicaux, peuples indigènes insurgés, victimes de massacres, tous les sacrifiés et les dignes morts du système, et puis les ancêtres glorieux de la littérature, Cernuda, Neruda, Revueltas, et puis les fictifs, les romanesques, tenanciers de cantina, de puesto, chauffeurs de taxi, travesti romantique, tous ceux dont la parole, toujours vive, toujours à l'emporte-pièce, s'inscrit dans la construction d'un autre projet du monde où le signe aurait à nouveau l'autorité sur les choses.

A l'image des espaces qu'il met en scène - monstrueuse capitale, selva re-sauvagisée par la force brute -, le roman s'affirme, non comme l'espace lisse d'une stéréotypie morte, mais à son tour, comme l'espace insurgé où s'énoncent tensions agoniques, fissures de sens et de syntaxe, et autres effractions poétiques ordinaires, ordurières, métaphoriques... Effractions de sons, de sens, fractures obliques - parodie, ironie, dialectique -, seules réponses semble-t-il aux infractions du pouvoir mexicain amateur de masques, seuls recours contre l'apathie routinière de la langue ou de la société civile, oppositions exemplaires à la rhétorique et au silence qui accompagnent aussi la barbarie... Se construit, à partir d'une multiplicité de figures pour l'essentiel défigurées et prises à contre-pied dans l'écriture, la physionomie spectrale d'un pouvoir abandonné aux barbares.

Ce qui s'énonce là n'est pas autre chose sans doute, que la foi en la qualité de l'homme, animal politique parce qu'animal littéraire sensible au 
pouvoir du langage (Rancière 2000: 65). Un pouvoir susceptible d'entrer en contagion avec le réel: de faire de la chair des mots, depuis l'infinité des trajectoires obliques et la perturbation de l'ordre générique et linguistique, une renaissance des valeurs du politique, autorité plutôt que pouvoir, et de cette renaissance la condition d'une reconnaissance, prolongeant ainsi le projet de l'EZLN.

Partition ludique sur la confusion du réel ou mélodie en sous-sol échappée de la selva chiapanèque, ce roman interroge avant tout l'enjeu de ce que nous pouvons désigner comme une po-éthique: cette brèche creusée dans la matière verbale pour précisément produire du politique en produisant du poétique, venerabilis miraculo litterarum dit Jacques Rancière. Et il ajoute, évoquant l'autorité: "L'auctor inspire le respect par un rapport prodigieux à la lettre, à ce qui se dit et s'écrit, à ce qui s'annonce et s'interprète au moyen des lettres. Il est celui qui sait discerner le sens dans le bruit du monde» (Rancière 2004). Aussi, en déplaçant - même maladroitement, même à l'excès - les représentations, en défaisant les locutions figées, le roman inverse le sens de l'horizon qui ne symbolise plus alors la clôture du monde, du corps ou du langage, mais, citant Michel Collot, «leur ouverture à l'infini du désir» (90).

\section{Bibliographie}

Aguirre Rojas, Carlos Antonio, 2003, Mitos y olvidos en la historia oficial de México, México, Quinto Sol.

Angenot, Marc, 1995, La parole pamphlétaire, Paris, Éditions Payot.

Arendt, Hannah, 1972, La crise de la culture, Paris, Éditions Gallimard.

Bhabha Homi K., 2007, Les lieux de la culture Une théorie postcoloniale, Paris, Éditions Payot \& Rivages.

Bourdieu, Pierre, 1980, Le sens pratique, Paris, Éditions de Minuit.

Bourdieu, Pierre, 2001, Langage et pouvoir symbolique, Paris, Éditions du Seuil.

Charaudeau, Patrick, 2005, Le discours politique, les masques du pouvoir, Paris, Vuibert.

Collot, Michel, 1983, La poésie moderne et la structure d'horizon, Paris, PUF.

Devalle Susana, 1989, La diversidad prohibida: resistencia étnica y poder de Estado, México, El Colegio de México.

Derrida, Jacques, 1972, La dissémination, Paris, Éditions du Seuil.

Hamon, Philippe, 1996, L'Ironie littéraire, Essai sur les formes de l'écriture oblique, Paris, Hachette Supérieur. 
Perrin, Laurent, 1996, L’ironie mise en trope, Du sens des énoncés hyperboliques et ironiques, Paris, Éditions Kimé.

Rancière, Jacques, 2000, Le partage du sensible, esthétique et politique, Paris, La Fabrique-Éditions.

Rancière, Jacques, 2004, Aux bords du politique, Paris, Gallimard.

Revault d'Allonnes, Myriam, 2006, Le pouvoir des commencements. Essai sur l'autorité, Paris, Éditions du Seuil.

Rosset, Clément, 1976, Le réel et son double, Essai sur l'illusion, Paris, Éditions Gallimard,

Ricœur, Paul, 1991, Du texte à l'action. Essais d’herméneutiques II, Paris, Points Essais.

Subcomandante Marcos, Taibo II, Paco Ignacio, 2005, Muertos incómodos (falta lo que falta), México, Joaquín Mortiz. 